
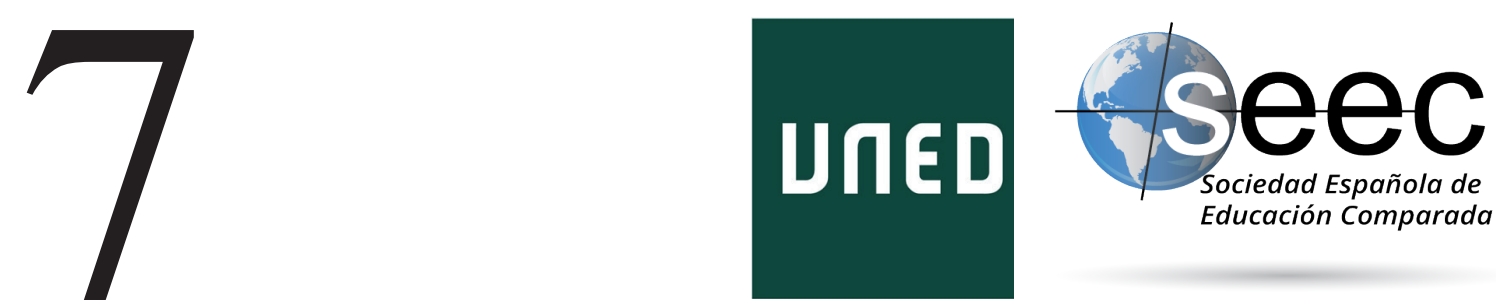

\title{
Los ciclos de reforma educativa en América Latina: 1960, 1990 y 2000
}

\author{
The cycles of educational reform in Latin \\ America: 1960,1990 and 2000
}

\section{Claudio Suasnábar*}

DOI: $10.5944 /$ reec.30.2017.19872

\author{
Recibido: 7 de octubre de 2017 \\ Aceptado: 22 de diciembre de 2017
}

\footnotetext{
* Claudio Suasnábar: Es Doctor en Ciencias Sociales y Magister en Ciencias Sociales con orientación en Educación (FLACSO/Argentina), Prof. en Ciencias de la Educación (Universidad Nacional de La Plata) y Post-doctorado en el Instituto de Educación de la Universidad de Lisboa. Actualmente se desempeña como Profesor Ordinario de Historia, Política del Sistema Educativo en la Universidad Nacional de La Plata. Es Investigador Principal del Programa Educación, Conocimiento y Sociedad del Área de la FLACSO. En los últimos años ha realizado diversas investigaciones en las áreas de política educativa, políticas universitarias y de educación superior e historia reciente del campo intelectual de la educación. Datos de contacto: E-mail: csuasnabar@gmail.com
} 


\title{
Resumen
}

Este artículo focaliza su interés en los ciclos de reforma educativa en América Latina entendidos como los distintos intentos estatales de reestructurar los sistemas educativos latinoamericanos, así como también las tendencias de cambio reciente en el gobierno de la educación y las nuevas formas de regulación a nivel mundial y regional. En esa dirección, la primera parte del texto analiza las reformas educativas de 1960 y los signos de agotamiento del modelo fundacional de los sistemas. La segunda explora los cambios en la matriz socio-política y las reformas educativas desarrolladas durante la década de 1990. La tercera se concentra en el nuevo escenario regional de comienzos del siglo XXI y las principales innovaciones políticas que introducen las reformas educativas de la década de 2000. Hacia el final se presentan una serie de reflexiones que procuran esbozar algunas claves del escenario educativo regional de los próximos años.

Palabras clave: América Latina; Reforma educativa; Política educativa; Gobierno de la educación

\begin{abstract}
This article focuses in the cycles of educational reform in Latin America understood as the different state attempts to restructure Latin American education systems, as well as the trends of recent change in the government of education and new forms of regulation worldwide and regional. In this direction, the first part of the text analyzes the educational reforms of 1960 and the signs of exhaustion of the foundational model of the systems. The second explores the changes in the socio-political matrix and educational reforms developed during the 1990s. The third focuses on the new regional scenario at the beginning of the twenty-first century and the main policy innovations that introduce the educational reforms of the 1990s. 2000. Toward the end, a series of reflections are presented that attempt to outline some of the keys to the regional educational scenario of the coming years.
\end{abstract}

Key Words: Latin America; Education Reform; Educational Policy; Government of education 


\section{Introducción}

Como señala Eric Hobbsbawm en su monumental estudio del siglo XX (1994), entre fines de la década de 1980 y los primeros años de la de 1990 termina una época del mundo y comienza una nueva. Así, la caída del Muro de Berlín y la posterior disolución de la URSS marca el final de aquella reducida centuria iniciada en la primera guerra mundial y que el historiador inglés denominará siglo XX corto.

No es casual, entonces que el clima de ideas de los años noventa estuvieran signados por una serie de intervenciones intelectuales que coincidían, por un lado, en proclamar (con mayor o menor optimismo) el final de un ciclo histórico, y por otro, procuraban dar cuenta de los rasgos y características de la nueva época. Quizás uno de los ensayos políticos más exitosos de aquellos años haya sido el trabajo de Francis Fukuyama («El fin de la historia») quién no dudaba en señalar el triunfo de la democracia liberal a escala mundial, el cual también constituía el punto final de la evolución ideológica de humanidad (1992). En rigor, la desintegración del bloque soviético no solo marcaba el fin de bipolaridad (capitalismo vs. socialismo) en la geopolítica mundial sino más bien expresaba la punta de un iceberg de tendencias de cambios en distintos órdenes, más profundas y de larga data.

Pasados casi treinta años, el escenario global se nos aparece bastante lejos del clima de triunfalismo y optimismo de comienzos de los noventa. Al contrario, la coyuntura actual se caracteriza por la crisis, la incertidumbre y fuertes signos de descomposición social que atraviesan tanto los países centrales como los periféricos. Así, la crisis de la economía mundial expresa no solo la desaceleración del crecimiento o virtual estancamiento sino también ciertas dinámicas centrífugas de «desglobalización» que se manifiestan en el retorno de discursos proteccionistas en el corazón mismo del capitalismo mundial. Consecuencia directa de estas tendencias es la creciente incertidumbre que rodea el orden político mundial cuya novedad principal lo constituye, por un lado, el desplazamiento del centro geopolítico de Occidente hacia Oriente (China y su zona de influencia), y por otro, estrechamente ligado a lo anterior, el debilitamiento de los bloques regionales y el resurgimiento del Estado Nacional. Por último, los fuertes signos de descomposición social ya no es un rasgo exclusivo del tercer mundo sino parte del paisaje de los países centrales que se revela en la expansión de ideologías conservadoras, nacionalista y xenófobas, y el ascenso de fuerzas políticas de extrema derecha y/o de populismos de derecha que ponen en riesgo la vitalidad de la democracia liberal de los países del Norte desarrollado (Sintomer, 2017).

Lejos de estar inmunes a estas tendencias, las sociedades latinoamericanas miran con recelo este escenario internacional signado por la crisis que por su magnitud resulta imposible no recibir sus consecuencias y efectos en un mundo globalizado.

Con todo, la primera década y media del nuevo siglo para América Latina constituyó un momento altamente favorable producto de la valorización de los productos primarios que exporta la región, lo cual posibilitó un inédito período de crecimiento económico. Asimismo, el fracaso de las reformas neoliberales de la década de 1990 y su secuela de crisis socio-económica no solo alimentaría la movilización y protesta social sino también estaría en el origen del ascenso de gobiernos de «izquierda», «nacional-populares» o «populistas» (según distintas perspectivas) pero que compartirían cierta orientación progresistas de las políticas impulsadas. La segunda mitad de la década de 2010 parecería marcar el agotamiento del llamado «boom de los commodities» y el ocaso de buena parte 
de estos gobiernos progresistas que, sin el impulso transformador de antaño se enfrentan con los límites y contradicciones internas de cada una de estas experiencias políticas. Al respecto, trabajos recientes como el de Leiras, Malamud y Stefanoni (2016) y Burchardt, (2017) analizan y discuten los diferencias y similitudes de los distintos casos nacionales.

En este escenario regional/global signado por la incertidumbre y una complejidad creciente deben ubicarse los problemas de los sistemas educativos y las políticas públicas para el sector, aunque - como veremos más adelante - buena parte de estos problemas se vinculan con tendencias de muy larga data que se superponen con los procesos en curso.

Quizás la problemática del gobierno de lo social-estatal sea aquella que revela con mayor claridad los puntos de contacto con las dificultades y complejidad que asume hoy el gobierno de la educación para enfrentar los problemas educativos. En este sentido, los sistemas educativos - como uno de los instrumentos claves de la conformación del Estado Nación- se erigieron alrededor de un modelo fundacional de organización burocrática cuyos rasgos principales estaban definidos por una estructura jerárquica y vertical, la diferenciación de roles y funciones, y un conjunto de normas y procedimientos. De tal forma, el gobierno del sistema educativo - entendido como la capacidad de direccionar y regular al conjunto de los actores e instituciones- descansaba, por un lado, sobre la homogeneidad y verticalidad de las acciones estatales, y por otro, sobre una clara diferenciación entre los que piensan la política (especialistas, técnicos, políticos) y los que ejecutan cotidianamente esa política (maestros y alumnos).

A inicios de la década de 1960, este modelo fundacional de los sistemas educativos y la forma de gobernar la educación antes mencionado, comenzaría a mostrar no solo deficiencias y limitaciones sino también claros signos de agotamiento. Las reformas educativas de esta década serán un primer intento de respuesta frente a estos problemas que luego de un impasse retomarán su impulso en la década de 1990 y de 2000 cuyas líneas de política expresan continuidades y rupturas.

En esta dirección, esta comunicación focaliza su interés en una mirada de largo plazo de los ciclos de reforma educativa entendidos como los distintos intentos estatales de reestructurar los sistemas educativos latinoamericanos, así como también las tendencias de cambio reciente en el gobierno de la educación y las nuevas formas de regulación a nivel mundial y regional. Esta noción de reforma educativa debe distinguirse analíticamente del concepto de innovación que remite a proyectos que procuran introducir modificaciones a nivel micro o local, y del concepto de cambio educativo que alude a las transformaciones que acontecen en distintas dimensiones de la educación formal: sistema, instituciones y aula. Así, los cambios pueden ser causados por reformas o innovaciones, pero también pueden acontecer independientemente de estos, por dinámicas internas y externas de los sistemas educativos (Popkewitz, 1991, Fullan, 2002 y Poggi, 2011)

Desde el punto de vista teórico, los ciclos de reforma educativa se inscriben en la problemática más amplia de los llamados policy transfer cuyos estudios han indagado la incidencia de diferentes actores, mecanismos y estructuras en la difusión de ciertas políticas (Dolowitz and Marsh, 2000). En el campo de la educación comparada los enfoques sobre policy borrowing y policy lending desarrollados por Gita Steiner-Khamsi (2004) y la noción de externalización de Jurgen Schriewer (1993 y 2001) nos aportan herramientas para comprender no solo los procesos de cambio y tendencias globales sino también permite captar las apropiaciones locales siempre marcadas por la cultura e historia de cada país o región. 
Ciertamente, la amplitud del período de análisis nos obliga a moderar nuestras pretensiones explicativas, razón por lo cual la noción de ciclos de reforma opera más bien como un recurso heurístico para «modelizar» procesos y tendencias, resaltando ciertos rasgos comunes de los casos nacionales. Asimismo, la noción de matriz socio-política (relación entre cierto tipo de Estado, modelo de desarrollo y régimen político-actores-sujetos) si bien no se desarrolla teóricamente opera como un supuesto la estrecha relación entre cambios en la matriz socio-política y los ciclos de reforma educativa en la región ${ }^{1}$.

Planteado de esta manera, el texto se organiza en cuatro secciones: la primera sección caracteriza los signos de agotamiento del modelo fundacional de los sistemas y las reformas educativas de 1960. La segunda sección explora los cambios en la matriz sociopolítica y las reformas educativas desarrolladas durante la década de 1990. La tercera sección se concentra en el nuevo escenario regional de comienzos del siglo XXI y las principales innovaciones políticas que introducen las reformas educativas de la década de 2000. Hacia el final se presentan una serie de reflexiones a modo de conclusiones que procuran esbozar algunas claves del escenario educativo regional de los próximos años.

\section{El agotamiento del modelo fundacional y las reformas educativas de la década de 1960}

Los sistemas educativos latinoamericanos desde sus orígenes se irían expandiendo y diversificando en las décadas con diferentes ritmos según los países. Hacia mediados del Siglo XX este patrón de desarrollo educativo comenzaría a dar muestras de agotamiento frente a las demandas y necesidades que planteaba el nuevo escenario internacional caracterizado por el auge de las ideologías del desarrollo. La «cuestión educativa» pasó entonces a un primer plano, considerada como pre-requisito para avanzar en el camino de transformación de las sociedades nacionales.

En rigor, la educación durante las décadas de 1960 y 1970 se convertiría en uno de los ejes centrales de la agenda de políticas públicas a nivel mundial que motorizaría procesos de reforma de los sistemas educativos en Europa y América Latina. Ciertamente, la simultaneidad de estos impulsos reformistas fueron en parte el resultado de la creciente influencia de la UNESCO y su prédica en favor de la expansión de la educación, y por otra, consecuencia de las diferentes coyunturas socio-económicas que atravesaban los países centrales y la periferia latinoamericana. En Europa la consolidación del Estado de Bienestar y el notable crecimiento económico estarán en el origen de las llamadas reformas educativas «comprehensivas» que supusieron la ampliación de la obligatoriedad escolar, la expansión diferenciada del nivel medio y el retraso de las instancias de selectividad (pasando de los 10 a 16-17 años). A su vez, esta progresiva universalización del nivel medio generó una mayor demanda por el acceso a la educación superior la cual también impulsó un proceso de diferenciación y diversificación de este nivel (Fernández Enguita y Levin, 1989).

En América Latina esta tendencia reformista se produjo en la década comprendida entre 1965 y 1975. Durante esos años varios países diseñaron e implementaron reformas en sus sistemas educativos como Chile en 1965, Honduras en 1966, Argentina y El Salvador

1 Para profundizar en estas nociones véase los siguientes trabajos: Garretón, Manuel Antonio (2002) La transformación de la acción colectiva en América Latina, en Revista de la CEPAL Nro. 76, Abril - Santiago de Chile, Acuña, Carlos (1995) La nueva matriz política argentina. Nueva Visión, Buenos Aires, y Cavarozzi, Marcelo (2000) El capitalismo político tardío y su crisis en América Latina. Homo Sapiens Ediciones, Rosario. (introducción) 
en 1968, Brasil en 1971, Perú, Costa Rica y Panamá en 1972 y México en 1973. Con diversos énfasis y logros en su implementación, las reformas educativas fueron concebidas como orgánicas o integrales por cuanto suponían cambios en la estructura del sistema orientadas principalmente a universalizar la educación primaria (reduciendo las desigualdades entre lo rural y lo urbano), modernizar los planes de estudio de este nivel y mejorar el sistema de formación de maestros.

En buena medida, la promesa del desarrollo que caracteriza el clima de optimismo de los años sesenta y setenta marcaría esta nueva etapa para la región, que encontrará en la idea de «modernización» aquella alternativa capaz de completar y corregir ese proceso contradictorio de ingreso a la modernidad de las sociedades latinoamericanas. Ciertamente, la fuerza persuasiva de esta amalgama de ideas conocidas como desarrollismo, radicaba en la convicción ampliamente extendida de la capacidad estatal para promover y orientar un proceso de cambio social entendido este como un proceso gradual y secuencial de cumplimiento de ciertas metas-objetivos (Suasnábar, 2004).

Esta racionalidad típicamente moderna se fortalecería con la institucionalización académica de las carreras de sociología, economía y ciencia política; las cuales rápidamente difundirían un conjunto de nuevas tecnologías de intervención social denominada «planificación económico-social» que sería la estrategia y herramienta central para el cumplimiento de las etapas del desarrollo (Gurrieri, 1987). Como parte de este mismo proceso, la difusión de las teorías de capital humano y de formación de recursos humanos en el campo educativo no solo darían cuenta del valor económico de la educación y la importancia estratégica de mejorar las calificaciones de la fuerza de trabajo sino también aportarían un conjunto de metodologías que conformarán el llamado «planeamiento educativo» (Fernández Lamarra y Aguerrondo, 1990).

No es casual entonces que desarrollo y modernización hayan sido las ideas-fuerza que motorizarán las reformas educativas iniciadas durante estas décadas. En sintonía con estas visiones se propusieron, por un lado, erradicar el analfabetismo, incorporar a la educación básica la población escolar excluida y ampliar la educación secundaria y, por otro lado, incorporar esta racionalidad «moderna» no solo a través de nuevas tecnologías de gobierno del sistema educativo, sino más profundamente en la concepción de la escuela ahora pensada como agente de cambio, la que supondría a su vez, la modernización del rol docente y las prácticas escolares. De tal manera, reformar los sistemas educativos se materializó en la idea de «más escuelas» y «más niños escolarizados» que guio la consigna de la época de garantizar la igualdad de oportunidades. Pese a que este último objetivo estuvo lejos de cumplirse, el ciclo de reformas educativas de los sesenta constituyó un período de fuerte expansión de la educación primera que achicarían las brechas entre los países de modernización temprana y aquellos de la zona andina y centroamericana tal como muestra el Cuadro 1. Así, en 1955 la distancia entre unos y otros grupos países variaba de 23 al $79 \%(+56)$ hacia comienzos de los noventa las distancias se habían reducido de 75 a $97 \%(+22)$. 
Cuadro 1. Porcentaje de población entre 14 y 17 años con educación primaria completa según años. Fuente. Elaboración propia sobre datos del SITEAL-UNESCO.

\begin{tabular}{|l|c|c|c|c|c|}
\hline \multirow{2}{*}{ Países } & \multicolumn{5}{|c|}{ Años } \\
\cline { 2 - 6 } & $\mathbf{1 9 5 5}$ & $\mathbf{1 9 6 5}$ & $\mathbf{1 9 7 5}$ & $\mathbf{1 9 8 5}$ & $\mathbf{1 9 9 5}$ \\
\hline Argentina & 79,9 & 85,0 & 91,1 & 95,8 & 97,4 \\
\hline Brasil & 52,8 & 67,9 & 78,0 & 83,1 & 88,8 \\
\hline México & 46,5 & 61,8 & 79,1 & 86,1 & 91,0 \\
\hline Chile & 65,5 & 82,1 & 89,1 & 94,5 & 97,5 \\
\hline Paraguay & 42,7 & 53,4 & 71,1 & 81,6 & 85,4 \\
\hline Bolivia & 23,8 & 39,5 & 53,4 & 62,5 & 79,0 \\
\hline Perú & 47,4 & 65,6 & 78,4 & 83,2 & 89,8 \\
\hline Costa Rica & 58,1 & 77,1 & 87,3 & $\mathrm{~s} / \mathrm{d}$ & 89,2 \\
\hline El Salvador & 27,4 & 42,2 & 56,4 & 64,1 & 75,1 \\
\hline
\end{tabular}

Ahora bien, si la expansión educativa («más escuelas» $\mathrm{y}$ «más niños escolarizados») materializaba la idea de desarrollo por oposición al atraso educativo, la propuesta de modernización que impulsarían las reformas también comenzaría a asociarse al cuestionamiento/ruptura con la «escuela tradicional».

Ciertamente, esta visión crítica no solo expresaba la toma de conciencia del agotamiento del modelo fundacional de los sistemas educativos, sino también de los profundos cambios que se estaban operando en la propia teoría educacional que se revelará en la mudanza en los patrones de producción, validación y legitimación del conocimiento sobre la educación. En este sentido, la acelerada institucionalización de la investigación educativa hacia mediados del Siglo XX, asociada a las tareas de la planificación educativa, marcará el surgimiento de un nuevo tipo de profesionales que se asentarán en las universidades, en las oficinas técnicas de los ministerios y en los organismos internacionales dedicados a la educación (Husén, 1988; Landshere, 1996). Esta academización del campo educativo si bien significaría un impulso notable para la producción de conocimiento, así como la renovación y difusión de nuevos enfoques teóricos, también produciría un progresivo distanciamiento entre la teoría educacional y las prácticas escolares (Novoa, 1998). Todos estos cambios convergerán ya no solo en el cuestionamiento a la escuela tradicional, sino particularmente en las concepciones sobre el rol docente y la enseñanza.

De tal modo, las reformas educativas de los sesenta redefinirían la tradicional función civilizatoria de la escuela, que sería ahora visualizada como motor de cambio, esto es, como agente de modernización que en algunos países se expresará en la expansión de la escuela asociadas al desarrollo rural, mientras que en otros impulsará la educación técnico-profesional que se suponía debía acompañar el cambio de las estructuras productivas. Las nuevas funciones de la escuela demandaban, a su vez, la propia redefinición del rol docente que comenzaría a tematizarse alrededor de la idea de profesionalización, que condensaría, por un lado, este nuevo mandato modernizador, y por otro lado, los saberes y conocimientos provenientes de las nuevas corrientes educacionales. No es casual, entonces, que una de las líneas de política impulsadas en estas décadas orientada hacia la generación de este «maestro moderno» haya sido el cambio en la formación docente que pasará al nivel superior en reemplazo de la anterior formación normalista. 
Las tendencias de cambio reseñadas permiten caracterizar las décadas de sesenta y setenta como un punto de inflexión de los sistemas educativos de la región, que bajo la consigna modernizadora introducirá cambios en las modalidades de intervención estatal a través de la planificación, difundirá nuevas corrientes del pensamiento educativo y marcará el surgimiento de nuevos profesionales, que en conjunto complejizarán las formas del regulación y gobierno del sistema. La escuela como agente de cambio y la modernización del rol docente se plantearán como objetivos de estas políticas, las cuales también constituyen un punto de inflexión en la configuración histórica de la docencia no solo por las nuevas funciones sino particularmente en la redefinición de la propia tarea pedagógica que introducirían la teoría educacional.

Pese a las innovaciones y la renovación del discurso pedagógico que introducirían las reformas, lo cierto es que el modelo fundacional del sistema no se modificó sustancialmente. Por ello, este período si bien desde el punto de vista de las ideas educativas y la profesionalización académica resulta un punto de inflexión, por otra parte, también expresa una fuerte continuidad de los rasgos estructurales del sistema y la cultura escolar que se manifiesta en una expansión desigual y una pauta de diferenciación social selectiva.

\section{Las reformas educativas de la década de 1990 y el cambio de la matriz socio-educativa}

Ciertamente, las décadas del cincuenta y sesenta fueron los «años dorados» del desarrollismo, donde este universo de ideas y propuestas no dejó de estar signado por una mirada optimista respecto de las posibilidades de cambio. Los primeros años de la década del setenta marcaría para la CEPAL, principal impulsora de estas ideas, una nueva etapa cuyo rasgo más importante será la pérdida de aquella capacidad de influir y generar debate que tanto la había caracterizado en los años precedentes. Diferentes factores están en la base de este momento de eclipse de las ideas del desarrollismo cepaliano: por un lado, la crisis petrolera de 1973 y la recesión mundial que la acompañaría se manifestó en América Latina a través de políticas de endeudamiento externo.

Esta reorientación de la economía mundial se daría en paralelo a la gradual declinación del keynesianismo y con ello también la decadencia de las teorías del desarrollo. Este cambio en el clima de ideas se manifestaría en la rápida aparición de la nueva ortodoxia neo-liberal que sería adoptada casi sin reservas en los países del cono sur, que impulsarían las dictaduras militares. Así, la conjunción de «crisis económica» y «crisis en las ideas» dejarían a la CEPAL marginada por casi una década del debate político y económico (Bielchowsky, 1998).

$\mathrm{Al}$ igual que en el conjunto de las ciencias sociales latinoamericanas, la irrupción de las dictaduras militares y el nuevo clima intelectual supondría un momento de revisión crítica de las perspectivas teóricas y de la experiencia política de aquellas décadas. En el caso de la CEPAL este movimiento se expresaría en una discusión alrededor de la propia idea de desarrollo y más profundamente en la toma de conciencia del fracaso de las experiencias de planeamiento. Esta revisión crítica estaráa revisimás profundamente de deas,crcr marcada por un desplazamiento de la idea de desarrollo a la noción de «estilos de desarrollo», y por un cambio en el campo problemático que se expresará en la introducción de nuevos conceptos provenientes de la sociología y la ciencia política (Suasnábar, 2013). 
En este contexto, el balance de la «década perdida» y el nuevo escenario internacional signado por la globalización e internacionalización de los mercados planteaba nuevamente el interrogante sobre el modelo de desarrollo que debería seguir América Latina. En este sentido, la propuesta impulsada por la CEPAL/UNESCO a comienzos de los noventa fue un intento de respuesta al nuevo escenario ubicando a la educación como el pilar de una estrategia de desarrollo que se sintetizo en la consigna «Educación y conocimiento: eje de la transformación productiva con equidad» (1992). La propuesta cepaliana partió de un diagnóstico del sistema educativo que en líneas generales era acertado por cuanto identificaba con claridad que la expansión escolar operada en las décadas anteriores, se había realizado a expensas del deterioro en la calidad de los aprendizajes, lo cual profundizaba una desigual distribución del bien educativo. Este diagnóstico sería recuperado por el Banco Interamericano de Desarrollo (BID) y el Banco Mundial (BM) pero resignificando las líneas e instrumentos de políticas en sintonía con las propuestas neoliberales de reforma estructural (Krawczy, 2002).

De tal manera, las reformas educativas de los noventa expresaron esta nueva promesa modernizadora que se materializó en una agenda de políticas específicas (descentralización, focalización, autonomía escolar, etc.) y en un conjunto de instrumentos (evaluación, currículum nacional, financiamiento por proyectos, etcétera) cuya modalidad de intervención en el sistema fueron los programas específicos que promovieron una llegada directa hacia las escuelas con poca o escasa mediación de las estructuras intermedias del sistema (Gajardo, 1999; Navarro, 2007; Kaufman y Nelson, 2005).

Este discurso reformista elaborado por expertos (Braslavsky y Cosse, 1996) pero asumido como propio por los políticos, se sustentaba en el supuesto tácito que la racionalidad que portaban aquellas políticas y estos instrumentos bastaban para producir una transformación profunda de la dinámica del sistema, y más aún de las prácticas escolares. Asimismo, el discurso de los expertos y su racionalidad en la implementación de estas reformas acentuó más el distanciamiento, tanto de los funcionarios políticos como de las escuelas y los docentes.

Este distanciamiento se manifestó en una fuerte academización del discurso educativo como en el caso de las visiones curriculares y las didácticas específicas promovidas por las políticas de capacitación docentes con las cuales se pensaba transformar las prácticas docentes. Así, la centralidad asignada a la renovación de los contenidos que motorizó el cambio curricular se sustentó en una concepción ciertamente ingenua que consideraba que la sola actualización de los contenidos «per se» sumado a la idea de que la «diseminación» de dichos contenidos vía la capacitación docente podría modificar los procesos de enseñanza y aprendizaje en aula. No es casual, que en el discurso reformista ambas líneas de política (curriculum y capacitación) alimentaran una visión utópica de las bondades de dichas intervenciones (Dussel, 2001 y Valliant, 2007).

Por su parte, las propuestas de autonomía escolar que impulsaron las reformas contribuyeron fuertemente al desdibujamiento del rol docente, por cuanto afectaba a los modos tradicionales de gestionar las escuelas. En rigor cabe señalar que salvo Chile las propuestas de autonomía escolar no tuvieron peso significativo en las experiencias del resto de los países. Más retórico que real, este discurso autonomista tendió a extrapolar las formas de gestión empresarial al de las escuelas e introducir una lógica competitiva de financiamientos por proyecto, la cual se volvía contradictoria con un tipo de regulación burocrática, jerárquica y centralizada que continuaba estructurando no solo la vida cotidiana de las escuelas sino también la propia profesión docente. En este sentido, 
las políticas de descentralización si bien se proponían aproximar las instancias de toma de decisión a los niveles inferiores del sistema, lejos de favorecer las propuestas de autonomía escolar terminaron acentuando las tendencias de fragmentación del sistema (Kaufman y Nelson, 2005; Navarro, 2006).

Durante la década de 1990 las dinámicas de expansión educativa a nivel regional se volverían más complejas y contradictorias ya que si, por un lado, el objetivo de universalizar la educación primaria parecía estar más próximo en un grupo de países que superaban o se aproximaban al 90 \% de conclusión de estos estudios, por otro lado, el nivel secundario como da cuenta el Cuadro 2 estaba lejos de aquella meta aun en los países de modernización temprana (oscilando entre 50 y 70 \%), mientras que en el restos seguía manteniendo el carácter elitista (entre 30 y 40 \%). Asimismo, focalizando en la comparación entre 1995 y 2005 vemos que las reformas tuvieron logros y avances dispares según los países que explican la persistencia de las brechas antes señaladas.

Cuadro 2. Porcentaje de población entre 19 y 22 años con secundaria completa según año-

Fuente. Elaboración propia sobre datos del SITEAL-UNESCO.

\begin{tabular}{|l|c|c|c|c|c|c|}
\hline \multirow{2}{*}{ Países } & \multicolumn{6}{|c|}{ Años } \\
\cline { 2 - 7 } & $\mathbf{1 9 5 5}$ & $\mathbf{1 9 6 5}$ & $\mathbf{1 9 7 5}$ & $\mathbf{1 9 8 5}$ & $\mathbf{1 9 9 5}$ & $\mathbf{2 0 0 5}$ \\
\hline Argentina & 24,5 & 31,6 & 43,2 & 49,0 & 54,3 & 63,5 \\
\hline Brasil & 15,7 & 23,9 & 32,2 & 40,9 & 48,8 & 52,1 \\
\hline México & 10,0 & 19,8 & 27,9 & 32,9 & 36,5 & 48,3 \\
\hline Chile & 22,6 & 34,2 & 48,7 & 58,2 & 71,2 & 77,2 \\
\hline Paraguay & 10,5 & 18,2 & 22,0 & 28,8 & 38,2 & 27,5 \\
\hline Bolivia & 13,8 & 22,2 & 30,2 & 30,0 & 45,8 & 54,6 \\
\hline Perú & 18,5 & 29,4 & 47,0 & 51,7 & 61,6 & 71,6 \\
\hline Costa Rica & 13,6 & 27,5 & 36,2 & s/d & 40,6 & 45,7 \\
\hline El Salvador & 10,3 & 16,5 & 21,7 & 29,4 & 36,2 & 37,8 \\
\hline
\end{tabular}

De manera general, podemos decir que las reformas de los noventa si bien incorporaron un conjunto de políticas específicas y nuevos dispositivos de gobierno su propia racionalidad técnico-pedagógica terminaron desdibujando una mirada estructural de los problemas educativos, hecho que se revela en que casi ninguno de los países modificó la arquitectura organizacional de las escuelas. Por ello es que el balance de las reformas de los noventa, si bien marcan un punto de ruptura respecto del período anterior, por otro lado, expresa las mismas limitaciones de una concepción tecnocrática que piensa el cambio educativo desde una visión restringida sin tomar nota de la complejidad que supone modificar las prácticas docentes y la cultura escolar.

En buena medida, la suerte de estas reformas educativas estuvo ligada al proceso de reconversión y reestructuración socio-económica que se dieron durante aquellos mismos años (Corrales, 1999). Así, para finales de la década el fracaso del consenso neoliberal que orientó estos procesos quedó en evidencia no solo por el aumento de la desigualdad y la profundización de la exclusión social sino también por la crisis de cohesión social e inestabilidad política en toda la región. No es casual, que en este contexto los logros y avances de las reformas educativas como el aumento de la tasa de escolarización de nivel secundario se diera en paralelo a una mayor fragmentación social y pérdida de calidad educativa. 


\section{Las reformas educativas deladécada de 2000: ampliación de derechos, experiencias nacionales y consolidación de nuevas formas de regulación}

Hacia la segunda mitad de la década del 2000 la decepción por los resultados de las evaluaciones de la calidad marca un punto de inflexión en los debates recientes de política educativa, el cual con distintas características en cada país se expresará en la emergencia de una nueva agenda de política (Iaies, 2011). Así, luego de más de una década de reformas la percepción de las administraciones educativas era la de una asimetría entre la inversión de recursos y los modestos resultados obtenidos, hecho que revela, a su vez, el bajo impacto de las políticas implementadas para modificar el comportamiento y las prácticas de los actores del sistema.

$\mathrm{Al}$ respecto, distintos estudios que realizan un balance de las reformas educativas de la década de 1990 parecen corroborar esta percepción de los gobiernos, que se sintetiza en la contradictoria expresión de «avances importantes para magros resultados» (Gajardo, 1999; Grindle, 2000; Kaufman y Nelson, 2005). Esta caracterización da cuenta que si bien, en la mayoría de los países creció la presencia de la educación en la agenda política nacional (sanciones de nuevas leyes de educación, procesos de descentralización, políticas focalizadas y participación en pruebas internacionales), las deudas acumuladas de las reformas superan estas acciones por cuanto constatan la persistencia de la brecha educativa entre países y estratos sociales no solo expresadas en el desigualdad en el acceso sino también en la distribución y apropiación del conocimiento.

Como señalamos al inicio, los primeros años de la década de 2000 marcaron un cambio de frente en la dinámica del capitalismo global expresado por el ascenso de China como motor principal de la economía mundial, hecho que ciertamente cambiará la suerte de América Latina. Así, entre 2003 y 2008 el gigante asiático crecerá a un promedio anual de 10 \% (las llamadas tasas «chinas») que se manifestará no solo en su transformación como potencia exportadora sino también por su demanda insaciable de materias primas que está en la base del «boom de los commodities» que experimentará la región. En paralelo, la llegada al gobierno de fuerzas o coaliciones de orientación progresista en distintos países fue una respuesta frente al fracaso de las reformas neoliberales de la década de 1990 y sus consecuencias de crisis socio-económica e inestabilidad política.

Este nuevo escenario signado por la bonanza económica ampliaría los márgenes de acción de la mayoría de los gobiernos que se manifestará en la recuperación del rol del Estado adoptando la forma de un neo-intervencionismo y el despliegue de una agenda de políticas públicas orientadas a reconstituir el tejido social y mejorar las condiciones de vida de la población.

En este sentido, las reformas educativas de la década de 2000 son parte de este conjunto de iniciativas de reparación social, pero a diferencia del ciclo anterior donde la agenda de política adquirió la forma de un "paquete de reforma» impulsado y/o promovida por los organismos internacionales, este ciclo parece más el resultado de la convergencia de diferentes (y a veces contradictorias) políticas educativas nacionales. Dicho de otra manera, la agenda de reforma es la reconstrucción, a posteriori de una serie de experiencias e iniciativas nacionales que serían difundidas, apropiadas y reformuladas en cada país en el marco del relanzamiento de los bloques regionales. 
El común denominador de estas experiencias y procesos lo constituye indudablemente la expansión de los derechos educativos ${ }^{2}$ que, a su vez se caracterizará por tres grandes rasgos: a) la ampliación del acceso a todos los niveles, b) el reconocimiento de derechos de poblaciones excluidas y marginadas, y c) el crecimiento notable del financiamiento educativo. Ciertamente, «el derecho a la educación» como demanda-consigna se gestó en la década anterior para movilizar y nuclear la resistencia frente a las reformas neoliberales. Esta retórica será recuperada y plasmada en un conjunto de nuevas leyes de educación que se sancionarían en estos años (Cuadro 3) que simultáneamente promoverán el acceso universal de la educación en todos sus niveles (incluida la educación superior), y extenderá el reconocimiento y derecho a la educación a grupos raciales, étnicos y/o de género antes excluidos, materializándose en declaraciones y en algunos casos con dispositivos concretos para garantizar estos derechos (López, 2007). Asimismo, estas nuevas leyes extenderán la obligatoriedad escolar que en promedio pasaría de 10 a 13 años.

Cuadro 3. Leyes nacionales de educación vigentes por país.

Fuente. Rivas, A. (2015) América Latina después de PISA. Lecciones aprendidas de la educación en siete países (2000-2015). Buenos Aires: CIPPEC-Natura-Instituto Natura.

\begin{tabular}{|l|c|c|c|}
\hline \multicolumn{1}{|c|}{ País } & Nombre de Ley & $\begin{array}{c}\text { Año de } \\
\text { Sanción }\end{array}$ & $\begin{array}{c}\text { Última } \\
\text { modificación }\end{array}$ \\
\hline Argentina & Ley de Educación Nacional (26.206) & 2006 & s.d \\
\hline Brasil & Ley de Directrices y Bases de la educación na- & 1996 & 2009 \\
\hline Chile & Ley General de Educación (20.370) & 2009 & \\
\hline Colombia & Ley General de Educación (1.151) & 1994 & 2013 \\
\hline México & Ley General de Educación (DOF 13-7-1993) & 1993 & 2014 \\
\hline Perú & Ley General de Educación (28.044) & 2003 & \\
\hline Uruguay & Ley General de Educación (18.437) & 2009 & \\
\hline
\end{tabular}

Esta expansión y ampliación de derechos educativos aconteció en el marco de un extraordinario ciclo de crecimiento económico, el cual no solo posibilitó un aumento del financiamiento educativo como nunca antes había acontecido sino también permitió una reducción significativa de la indigencia, la pobreza y el desempleo, aunque dicho impulso

2 Nos referimos a «derechos educativos» siguiendo la conceptualización socio-política de Axel Rivas quién señala que: «En muchos sentidos, los distintos derechos educativos analizados conforman un horizonte no alcanzado aún por los marcos legales vigentes, que si bien protegen en muchos aspectos el derecho a la educación, todavía requieren numerosas adaptaciones. Por eso, la visión sociopolítica del derecho a la educación no descansa en la legalidad vigente, sino que se inscribe en un paradigma de acción para alcanzar nuevas conquistas sociales, capaces de ser traducidas en normas específicas» (Rivas, 2007: p. 7). 
no alcanzaría para modificar la desigualdad estructural de las sociedades latinoamericanas. Como se puede observar en el Cuadro 4, los países de la región en 2000 gastaban en educación un promedio de 4,3\% del PBI, el cual pasaría al 5,0 \% en 2008. Este crecimiento global de los recursos destinados a la educación encubre esfuerzos y puntos de partida diferentes de cada uno de los países, situación que se puede apreciar en la última columna del cuadro donde Brasil (sacando el caso excepcional de Cuba) muestra uno de los mayores procesos de inversión. Otro dato interesante es que comparado con el promedio del gasto público en educación de la Unión Europea de 5,3 \% (2012)3, varios países se aproximan e incluso superan esta inversión.

Cuadro 4. Gasto Público en Educación como porcentaje de PBI (200o-2008).

Fuente. Albornoz y Warnes (2013) Educación en América Latina: más gasto, ¿̇mismos resultados?, en Foro Económico http://focoeconomico.org/2013/o1/23/educacion-en-americalatina-mas-gasto-mismos-resultados/.

\begin{tabular}{|l|c|c|c|}
\hline \multicolumn{1}{|c|}{ País } & $\mathbf{2 0 0 0}$ & $\mathbf{2 0 0 8}$ & Variación Total \\
\hline Argentina & 4,6 & 5,4 & 17,3 \\
\hline Brasil & 4,0 & 5,4 & 34,6 \\
\hline México & 4,9 & 4,9 & 0,5 \\
\hline Chile & 3,9 & 4,0 & 2,0 \\
\hline Uruguay & 2,4 & 2,9 & 18,9 \\
\hline Colombia & 3,5 & 3,9 & 84,6 \\
\hline Cuba & 7,7 & 14,0 & 46,9 \\
\hline El Salvador & 2,5 & 3,7 & 14,7 \\
\hline Costa Rica & 4,4 & 5,0 & 6,4 \\
\hline Promedio General & 4,3 & 5,0 & \\
\hline Promedio sin Cuba & & & \\
\hline
\end{tabular}

El impacto de la ampliación de derechos y el aumento del financiamiento educativo fue el motor en un nuevo ciclo de expansión matricular en la región que se concentrará en el nivel secundario y en menor medida en el nivel inicial ${ }^{4}$. De tal manera, si hasta

3 Eurostat. "Educational expenditure statistics" - Statistics Explained (2/11/2017) http://epp. eurostat.ec.europa.eu/statistics_explained/index.php/Educational_expenditure_statistics\#

4 Para una mirada comparada sobre las principales reformas y tendencias de cambio del nivel 
mediados del siglo XX la escuela media era una institución elitista donde pocos países superaban el $20 \%$ de su población de 19 a 22 años con secundaria completa, para 2005 la mitad de los países supera el 50 \% lo cual da cuenta del proceso de masificación de este nivel educativo (Cuadro 2).

Con todo, los últimos 10 años dejaran en evidencia los límites de este proceso de expansión que se manifiesta en la brecha entre acceso y conclusión de los estudios tal como lo muestra el Cuadro 5. Así, las tasas de escolarización (bruta y neta) nos indican el grado de acceso y asistencia a un determinado nivel escolar. En el caso de la escuela media la comparación entre 2000 y 2010 nos muestra una ampliación significativa del acceso en algunos países como Paraguay, Bolivia, Perú y Costa Rica (crecen $+10 \%$ ), mientras que el resto está por debajo y algunos como Argentina aumentó solo un $3 \%$ $(\mathrm{TNE} 2000=81,4 ; 2010=84,5)$. Ahora bien, si analizamos la población de 19 a 22 años que concluyó sus estudios secundarios entre 2000 y 2010 vemos también un crecimiento importante, aunque por debajo de las tasas de escolarización, precisamente la última columna muestra en porcentajes esa diferencia entre acceso y conclusión. Para el final de la década ese diferencial se encontraba por encima del $30 \%$ salvo algunas excepciones como Chile con $17 \%$.

Asimismo, los límites de este proceso de expansión también se observan en la persistencia de las desigualdades sociales al interior y entre los países. Al respecto, algunos especialistas consideran que la dificultad para avanzar en la meta de universalizar el nivel secundario no es otra que el agotamiento y/o final de un modelo de expansión educativa que ya no es eficaz para incorporar a las aulas una heterogénea diversidad de grupos sociales.

Cuadro 5. Tasa bruta de escolarización (TBE), tasa neta de escolarización (TNE), porcentaje de jóvenes de 19 a 22 años con secundario completo según años. Fuente. Elaboración propia sobre datos de Izcovich, Gabriela (2014) La expansión educativa en el nivel medio. América Latina 2000-20010. Cuaderno 19, SITEAL-IIPE-UNESCO, Buenos Aires.

\begin{tabular}{|l|c|c|c|c|c|c|c|c|}
\hline \multirow{2}{*}{ Países } & \multicolumn{4}{|c|}{ Año 2000 } & \multicolumn{4}{c|}{ Año 2010 } \\
\cline { 2 - 9 } & TBE & TNE & $\begin{array}{c}\text { Porcentaje } \\
\text { 19 a 22 } \\
\text { secundario } \\
\text { completo }\end{array}$ & $\begin{array}{c}\text { Diferencia } \\
\text { entre } \\
\text { acceso y } \\
\text { conclusión }\end{array}$ & TBE & TNE & $\begin{array}{c}\text { Porcentaje } \\
19 \text { a 22 } \\
\text { secundario } \\
\text { completo }\end{array}$ & $\begin{array}{c}\text { Diferencia } \\
\text { entre } \\
\text { acceso y } \\
\text { conclusión }\end{array}$ \\
\hline Argentina & 91,6 & 81,4 & 53,7 & 37,9 & 92,9 & 84,5 & 60,1 & 32,8 \\
\hline Brasil & 89,4 & 70,2 & 40,1 & 49,3 & 92,2 & 77,6 & 53,7 & 38,5 \\
\hline México & 74,3 & 63,7 & 32,2 & 42,1 & 78,8 & 71,9 & 48,3 & 30,5 \\
\hline Chile & 93,1 & 77,6 & 59,8 & 33,3 & 94,9 & 81,7 & 77,2 & 17,7 \\
\hline Paraguay & 75,9 & 54,0 & 42,4 & 33,5 & 85,8 & 70,6 & 56,1 & 29,7 \\
\hline Bolivia & 83,2 & 61,8 & 43,0 & 40,2 & 91,3 & 75,3 & 53,2 & 38,0 \\
\hline Perú & 81,5 & 58,9 & 60,0 & 21,5 & 90,4 & 76,3 & 70,4 & 20,0 \\
\hline Costa Rica & 74,3 & 49,3 & 34,2 & 40,1 & 89,0 & 65,1 & 48,3 & 40,7 \\
\hline El Salvador & 65,8 & 50,1 & 32,8 & 33,0 & 72,9 & 57,1 & 39,6 & 33,3 \\
\hline
\end{tabular}

secundario en América del Sur véase Ruiz, G. (2016): Secondary Education in Five South American Countries. Revista Española de Educación Comparada. Sobre la expansión reciente del nivel inicial véase Izcovich, G. (2013) La expansión educativa en el nivel inicial durante la última década. Cuaderno 16, SITEAL-IIPE-UNESCO, Buenos Aires. 
Ligado a lo anterior, no es casual que las políticas contra las desigualdades se convertirán durante la década de 2000 en un verdadero laboratorio de innovación de políticas sociales que acompañaría la expansión de derechos educativos. Con eje en los Programas de Transferencias Condicionadas (PTC) ${ }^{5}$ que se expandirían por la región en lo peor de la crisis económica de fines de los noventa, estos programas contendrían la asistencia escolar como condicionalidad tal como se plantea en la Asignación Universal por Hijo (AUH) de Argentina, la Bolsa Familia de Brasil, el Oportunidades en México, Chile Solidario de este país y el programa Más Familias en Acción de Colombia, entre otros (SITEAL, 2016 y Cena, 2015). Este tipo de programas marcará una tendencia diferente de la década de 1990 cuyo rasgo dominante fueron los programas focalizados o compensatorios, mientras que en este caso se plantean como garantes del cumplimiento de derechos (universalistas), y a su vez, se desplazan de lo asistencial hacia lo educativo en una visión que procura ser más integral (Cecchini, 2014).

Paralelo a estas grandes iniciativas, los programas de becas y de extensión de la jornada escolar fueron las estrategias dominantes para incluir y retener a los alumnos que siguieron los países con diferentes resultados. En menor medida se cuentan la creación de ofertas alternativas o complementarias a las escuelas comunes para atender problemáticas específicas como sobreedad, abandono o diversidad cultural.

Durante la década de 2000 las transformaciones curriculares siguieron siendo (como en los noventa) un eje central de las políticas de reforma en la región. Al respecto, si bien la especificidad del curriculum excede las posibilidades de este artículo, es factible señalar como rasgo común de las experiencias nacionales la hibridez curricular, esto es, la convergencia entre cierto retorno a los contenidos disciplinares y asignaturas escolares que convive con curriculums más contextualizados e integrados, así como también entre un mayor o menor nivel de prescripción y autonomía de la práctica pedagógica. Asimismo, la presión de los sistemas de evaluación sobre las políticas curriculares se manifiesta en la generación de estándares esperados de aprendizaje y el diseño de curriculums por competencias en varios países (Dussel, 2006 y Rivas, 2015).

La distribución masiva de computadoras por alumno y la multiplicación exponencial de los materiales digitales marcan el gran salto tecnológico de la región durante esta década. Si bien fueron Uruguay, Argentina y Perú los países que avanzaron en el modelo 1 a 1 (una laptop por niño) ${ }^{6}$, la fuerza transformadora de las nuevas tecnologías se manifestó en toda la región en la expansión de portales educativos en Internet, creación de contenidos para formatos digitales, desarrollo de distintos softwares educativos y aplicaciones, programas de capacitación docente a grandes escalas, entre otros materiales (IIPE-UNESCO, 2014). Paralelamente a estos cambios, el crecimiento de la producción y distribución de libros de texto también debe contarse como la continuidad de una tendencia iniciada en los noventa, pero con larga tradición en la región. En este sentido, en México, Chile y Brasil desde mitad del siglo XX la distribución de textos escolares para cada niño se transformó en una política pública.

La expansión de los sistemas nacionales de evaluación y la mayor discusión sobre la calidad de la educación constituyen dos tendencias que si bien no son nuevas - ya que comenzaron con las reformas anteriores-, durante la década de 2000 las evaluaciones

$5 \quad$ Los PTC son programas consistentes en la distribución de dinero a condición de cumplir ciertos requisitos como puede ser la asistencia escolar y los controles de salud.

6 En 2007 Uruguay comienza con el Plan Ceibal y Perú con el Programa Huascarán, mientras que Argentina en 2010 se suma con el Plan Conectar Igualdad. 
estandarizadas se volverán dispositivos centrales en la regulación y gobierno de los sistemas educativos en la mayoría de los países de la región. Así, el pasaje de evaluaciones de calidad por muestras de escuelas hacia pruebas censales para toda la población escolar (o de ciertos años) transformó la evaluación en un dispositivo de alto impacto sobre la dinámica de los sistemas y el comportamiento de los actores. La mayor visibilidad de los resultados educativos y su difusión por medio de rankings o escalas amplificados por los medios de comunicación no solo instaló el problema educativo en la agenda de políticas sino también se volvió un factor de presión sobre los docentes y escuelas.

Un rápido repaso por las distintas experiencias nacionales nos muestra que Chile fue primer país en introducir sistemas de evaluación a través del SIMCE (Sistema de Medición de la Calidad de la Educación) creado en 1988 durante la dictadura militar de Pinochet, continúo usándose con el retorno a la democracia y recién en los 2000 una comisión de expertos introdujo grandes cambios y mejoras técnicas. Por su parte, Brasil tiene una larga experiencia en sistemas de evaluación, aunque centrado en el nivel superior. En 1990 crea el SAEB (Sistema de Avaliação de Educação Básica) de tipo muestral, el cual será el antecedente de la Prova Brasil creada en 2005 (censal) para evaluar a todos los alumnos de $5^{\circ}$ y $9^{\circ}$ año de la educación básica. La expansión de estos sistemas permitirá el desarrollo de una de las innovaciones más importante en términos de instrumento de política como es el IDEB (Índice de Desenvolvimiento da Educação Básica) que combina los resultados de la Prova Brasil con la trayectoria interna de los alumnos (flujo escolar). De esta manera, el IDEB traduce en términos de puntaje la relación entre retención y logros asignando mejor puntaje a las escuelas con alta retención y logros de aprendizaje obtienen. A su vez, el IDEB puede utilizarse a diferentes escalas comenzando por la sección de clase, escuela, ciudad, región, provincia y Estado Nacional, convirtiéndolo un gran "panóptico» de flujos y avances del sistema educativo.

En México los sistemas de evaluación son más recientes tales como el EXCALE (Exámenes de la Calidad y el Logro Educativos) creado en 2005 cuyo objetivo es medir los aprendizajes a nivel sistémico y por Estados (muestral) y el ENLACE (Evaluación Nacional del logro Académico en Centros Escolares) creado en 2006 para obtener información por escuela de los resultados escolares (censal). Argentina y Colombia también la evaluación de resultados de aprendizaje comienza en los noventa: en el caso argentino a partir del ONE (Operativo Nacional de Evaluación) fue creado en la década de 1990 y desde 2007 pasó a ser censal para los alumnos del último año del nivel secundario, mientras que para el caso colombiano inicia con la prueba SABER creada en 1991 (muestral) que en 2001 pasará a ser censal en distintos años de la educación básica.

Esta expansión y sofisticación de los sistemas de evaluación también se explica por el impacto de las pruebas internacionales (PISA) que desde 2000 comenzará a desarrollar la OCDE. Al principio muy criticadas y resistidas en la región, estimularía el desarrollo de experiencias propias a través del Laboratorio Latinoamericano de Evaluación de la Calidad Educativa (LLECE) de UNESCO que elaborará las pruebas SERCE (2006) y TERCE (2013). La progresiva visibilidad y resonancia política internacional de las pruebas PISA marcaría un progresivo acercamiento de los países de la región que ya para el segundo estudio de 2003 se incorporan Uruguay, México y Brasil, en 2006 se sumaran Chile, Colombia y Argentina, en 2009 se incorporaron Perú y Costa Rica, y finalmente en 2012 se agregaron Trinidad y Tobago y Republica Dominicana. 
Por último, el gobierno de los sistemas educativos fue otro de los grandes cambios operados durante la década de 2000. En este sentido, los procesos de descentralización impulsados por las reformas educativas de 1990 lejos de mejorar el gobierno generaron nuevos problemas asociados a la delegación de funciones hacia autoridades locales con limitadas capacidades y en algunos casos profundizaron la fragmentación del sistema y las desigualdades educativas. Asimismo, el bajo impacto de estas reformas también se explica por la modalidad de intervención consistente en un prolífico set de programas (orientados a distintos problemas), los cuales paradojalmente produjeron una creciente «opacidad» de la política para los actores escolares y una consecuente pérdida de legitimidad y eficacia (Iaies, 2011).

Frente al fracaso de estas iniciativas y en el marco de la recuperación de la centralidad del Estado del nuevo siglo comienza, por un lado, un acelerado proceso de recentralización de las políticas educativas, y por otro, se consolidan las nuevas formas de regulación introducidas en la década de 1990. De tal manera, a nivel del gobierno de la educación se produce una superposición híbrida entre ambas modalidades de intervención cuyo rasgo dominante es la contradictoria convivencia entre lógicas y racionalidades diferentes. Así, la tendencia recentralizadora que se manifestará en el retorno de las tecnologías de planificación de largo plazo y en visiones más integrales de las políticas (Betancur, 2011), mientras que simultáneamente los sistemas nacionales de evaluación y las pruebas estandarizadas consolidan el lugar de dispositivos de regulación vía control por resultados. Como señalamos anteriormente, la evaluación se vuelve un factor de presión que tiende a conducir y orientar no solo el comportamiento de los actores sino también la concretatización del curriculum escolar.

\section{A modo de conclusiones}

La segunda mitad de la década de 2010 parecería marcar el cierre del último ciclo de reformas educativas en la región. Al menos dos factores inciden en esta percepción, por un lado, el retorno de la austeridad económica producto del agotamiento del «boom de los commodities», y por otro, el ocaso de buena parte de los gobiernos progresistas (de izquierda, nacional-populares o populistas) y el ascenso de fuerzas políticas de derecha (tradicional, neo-liberal, gerencial o nueva derecha según distintas perspectivas). De tal manera, sea por el contexto de escases de recursos financieros como por el cambio de orientación política en algunos casos y la pérdida del impulso transformador en otros, lo cierto es que son signos claros de la finalización de un ciclo, aunque todavía no se perfile una nueva agenda de política educativa ni tampoco el inicio de un nuevo ciclo de reformas en la región.

En este sentido, si bien la coyuntura de nuestro tiempo presente (octubre de 2017) está signada por la incertidumbre del futuro, la mirada de largo plazo de tendencias y procesos de cambio en los sistemas educativos nos posibilita ensayar algunos análisis que, a modo de conclusiones, procuran esbozar algunas claves del escenario educativo regional de los próximos años.

Un primer aspecto remite al devenir de las tendencias y dinámicas de expansión educativa en la región. Al respecto, el peso social del discurso de la ampliación de derechos plasmado en las nuevas leyes de la década de 2000 ha sido y seguirá siendo un motor que alimente la demanda educativa y políticas orientadas a la inclusión social. Por ello es factible pensar que, aunque más ralentizado, las tendencias expansivas continúen siguiendo la 
dinámica estructural de una pauta de selección socio-educativa que consiste básicamente en que el valor democratizador del acceso masivo a un determinado nivel educativo se contrarresta con una diferenciación social de la oferta escolar (fragmentación educativa) y una distribución desigual del conocimiento (circuitos diferenciados de calidad).

Ciertamente, esta pauta de selección no es nueva, sino que constituye una de las funciones sociales que cumplen los sistemas educativos como parte de los dispositivos de gobierno en las sociedades modernas. Por ello no es casual que la educación haya sido históricamente una arena de disputa donde se dirime la lucha por el poder y el conflicto social en términos de acceso (quiénes entran en la escuela), distribución (qué saberes se trasmiten) y apropiación del conocimiento (qué capacidades y posibilidades generan). El desigual y en algunos casos incompleto ingreso a la modernidad de los países latinoamericanos está en el origen de las diferentes trayectorias históricas de los sistemas educativos que como vimos en anteriores apartados hacia mitad del siglo XX aquel modelo fundacional comienza a mostrar signos de agotamiento, esto es, los sistemas educativos ya no generan gobernabilidad en términos legitimidad y consenso de una determinada pauta de selección social.

Desde esta perspectiva de largo plazo, los ciclos de reforma educativa de 1960, 1990 y 2000 pueden ser pensados como coyunturas particulares de la región que ponen de manifiesto el grado y profundidad de la disputa socio-política por el acceso, distribución y apropiación del conocimiento.

Un segundo aspecto estrechamente ligado al anterior lo constituyen las tendencias de cambio del gobierno de la educación. Desde sus orígenes los sistemas educativos se conformaron alrededor de un modelo de organización burocrática cuyos rasgos principales estaban definidos por una estructura jerárquica y vertical, la diferenciación de roles y funciones, y un conjunto de normas y procedimientos. Así, el gobierno del sistema educativo entendido como la capacidad de direccionar y regular al conjunto de los actores e instituciones descansaba, por un lado, sobre la homogeneidad y verticalidad de las acciones estatales; y por otro, sobre una clara diferenciación entre los que piensan la política (especialistas, técnicos, políticos) y los que la ejecutan cotidianamente (maestros y alumnos).

Esta modalidad de gobierno que podemos caracterizar burocrático-centralizada también entra en crisis hacia mitad del siglo XX como consecuencia de la progresiva expansión y diferenciación de los sistemas educativos, así como por la complejización de sus funciones sociales. Frente a esta situación, cada uno de los ciclos de reforma educativa introdujo cambios en la estructura de gobierno, en los instrumentos de política y en las modalidades de intervención. Así, la reformas de 1960 procuraron incorporar una racionalidad moderna no solo a través de nuevas tecnologías de gobierno como la planificación educativa y la academización de los saberes pedagógicos sino también de pensar a la escuela como agente de cambio para el desarrollo. Las reformas educativas de 1990 a tono con el ideario neoliberal de retiro del Estado marcaron un cambio sustantivo en el gobierno de la educación a través del mix de política formado por: descentralización, autonomía escolar y evaluación de la calidad (y en algunos países se agregó el pasaje del financiamiento de la oferta a la demanda educativa). Este cambio se manifestará en un progresivo desplazamiento del tipo de gobierno burocrático-centralizado hacia un tipo de regulación pos-burocrática de control a distancia y por resultados ${ }^{7}$. Con todo,

$7 \quad$ Para esta caracterización nos apoyamos en el trabajo de Barroso (2005) sobre el concepto de regulación y su incidencia en el cambio de las políticas públicas, y el trabajo de Mahon (2008) sobre la emergencia y conceptualización de las modalidades de regulación post-burocrática en Europa. 
el modelo tradicional de gobierno sobrevivió a estos cambios porque no se tocaron la estructura organizacional del sistema (por ej.: estatutos docentes, gestión escolar), esto es, el conjunto de reglas (formales e informales), normas y principios que enmarcan la acción y comportamiento de los actores.

Las reformas educativas de 2000 en el marco de la recuperada centralidad del Estado iniciarían un movimiento inverso de recentralización de las políticas educativas, y a la vez, de consolidación de las nuevas formas de regulación introducidas en la década anterior. Así, el rasgo dominante del gobierno de la educación lo constituye la superposición híbrida entre ambos movimientos y modalidades de intervención (burocrática-centralizada vs. regulación pos-burocrática) que conllevan lógicas y racionalidades diferentes, las cuales obviamente limitan la capacidad de orientar y regular el comportamiento de los actores e instituciones del sistema. En este escenario paradójicamente uno de los dispositivos más antiguos y constitutivos de la escuela moderna como es la evaluación emerge como modalidad de gobierno posible (Barroso y Carvalho, 2011).

Lejos de ser una particularidad latinoamericana, el gobierno de los sistemas educativos constituye un problema y un desafío global para las políticas educativas que conlleva la necesidad de revisar críticamente las herramientas teórico-conceptuales, los instrumentos de política y modalidades de intervención.

Un tercer y último aspecto remiten a una serie de líneas de políticas que, si bien se inician en la última década, parecerían proyectarse hacia adelante como parte de una posible agenda, aunque esta todavía no resulta perceptible. Estas líneas de política se proponen como respuesta frente a los problemas educativos pendientes y los desafíos que plantea el siglo XXI. Ciertamente, las situaciones nacionales están marcadas por tipos de desarrollo educativo deferentes y coyunturas particulares, sin embargo, a nivel de los gobiernos parecería haber cierto consenso sobre la importancia de cada problemática, aunque no haya acuerdo respecto del qué (contenido) de estas políticas, el cómo implementarlas y con qué recursos financiarlas.

1) La universalización de la educación infantil es quizás el consenso más extendido y más antiguo por cuanto estuvo presente en las innumerables declaraciones de la UNESCO y otros organismos internacionales. En la última década y media se produjo un avance importante, aunque insuficiente y muchos países tienen como meta el acceso de toda la población escolar.

2) La educación secundaria como problemática educativa parece orientarse en dos líneas de política: por un lado, la deuda pendiente del ciclo anterior de lograr la universalización del acceso al nivel y, por otro lado, el desafío futuro de repensar los formatos escolares, esto es, una reforma desde adentro del nivel.

3) La cuestión docente constituye un ejemplo claro de alto consenso sobre su importancia y bajo acuerdo respecto de las políticas específicas. En rigor, lo que denominamos cuestión docente supone distintas dimensiones del problema tales como la formación docente inicial (acceso, curriculum e instituciones), las condiciones de trabajo (sistemas de ingreso, ascenso y salarios) y la formación docente continua (capacitación, especialización y profesionalización). 
4) La evaluación de los aprendizajes vía pruebas estandarizadas se consolida como línea de política en la región, aunque no está claro si este dispositivo supondrá mayor capacidad del nivel central para regular y orientar el comportamiento de los actores.

5) Las tecnologías en educación constituyen otro gran interrogante para las políticas educativas en la próxima década. En primer lugar, el vertiginoso cambio tecnológico plantea un horizonte de incertidumbre y, en segundo lugar, todavía es limitado el conocimiento respecto del impacto en la cultura escolar, así como también del modelo pedagógico capaz de apropiarse de la potencialidad de las tecnologías en la escuela.

Las cinco líneas de política mencionadas obviamente no agotan la larga lista de nuevas y viejas problemáticas educativas de la región tales como educación técnica, educación de adultos, educación especial, educación superior, el financiamiento educativo, la relación educación-desarrollo económico, entre otros. Más bien este recorte se plantea como una suerte de hipótesis de trabajo de una serie de tendencias de política que se proyectan en el escenario futuro que se nos aparece menos favorable que la década pasada, aunque no tan catastrófico como la de fines del siglo XX. La moneda todavía está en el aire.

\section{Bibliografía}

ACUÑA, C. (1995): La nueva matriz política argentina (Buenos Aires, Nueva Visión)

ALBORNOZ, F. y WARNES, P (2013) Educación en América Latina: más gasto, ¿mismos resultados?, en Foro Económico http://focoeconomico.org/2013/o1/23/ educacion-en-america-latina-mas-gasto-mismos-resultados

BARROSO, J. (2005): O Estado, a Educaçao e a regulaçao das políticas publicas, en Educaçao e Sociedade Vol. 26, Nro. 92, Especial - Out. Campinas. http://www. scielo.br/pdf/es/v26n92/v26n92ao2.pdf

BARROSO, J. e CARVALHO, L. M. (2011): Apontamentos sobre os «novos modos de regulação» à luz de estudos sobre as relações entre conhecimento e política», en Propuesta Educativa Número 36, Año 20, Nov, Vol 2, Págs. 9 a 24. http:// propuestaeducativa.flacso.org.ar/archivos/dossier_articulos/56.pdf

BETANCUR, N. (2011): Los Planes Nacionales de Educación en América Latina: ¿instrumentos para una nueva generación de políticas educativas? Material preparado para el XIV Curso Regional sobre Planificación y Formulación de Políticas Educativas IIPE-UNESCO, Bs. As., agosto.

BIELSCHOWSKY, R. (1998): Evolución de las ideas de la CEPAL, Revista de la CEPAL, Número Extraordinario Octubre. (Santiago de Chile, CEPAL) pp. 21-46

BRASLAVSKY, C. y COSSE, G. (1996): Las actuales reformas educativas en América Latina: Cuatro Actores, Tres Lógicas y Ocho Tensiones (Santiago de Chile, PREAL). 
BURCHARDT, H-J. (2017): La crisis actual de América Latina: ¿causas y soluciones?, Revista Nueva Sociedad, 267, enero-febrero. (http://nuso.org/media/articles/ downloads/8._TC_Burchardt_267.pdf ) consultado 6 de octubre 2017

CAVAROZZI, M. (2000): El capitalismo político tardío y su crisis en América Latina. (Rosario, Homo Sapiens Ediciones)

CENA, R. (2015): Programa de transferencia condicionadas de ingresos y Asignación Universal por Hijo para la protección social ¿una ruptura en términos de política social? en SABER. Revista Multidisciplinaria del Consejo de Investigación de la Universidad de Oriente, vol. 27, núm. 4, octubre-diciembre, pp. 609-616

CECCHINI, S. (2014): Educación, Programas de Transferencias Condicionadas y protección social en América Latina y el Caribe, en FEIJO, M. y POGGI, M. (coord.) Educación y Políticas Sociales para la Inclusión, pp. (Buenos Aires, IIPE - UNESCO).

CORRALES, J. (1999): Aspectos Políticos en la Implementación de las reformas educativas, Documento $\mathrm{N}^{\circ}$ 14, (Santiago de Chile, PREAL).

DOLOWITZ, D. and MARSH, D. (2000): Learning from Abroad: The Role of Policy Transfer in Contemporary Policy-Making. Governance, 13:5-23.

DUSSEL, I. (2001): Las políticas curriculares de la última década en América Latina: nuevos actores, nuevos problemas. Extraído de http://www.oei.es/ reformaseducativas/politicas_curriculares_ultima_decada_AL_dus sel.pdf

DUSSEL, I. (2006): Estudio sobre gestión y desarrollo curricular en países de América Latina.Ponencia presentada en la Segunda Reunión del ComitéIntergubernamental del Proyecto Regional de Educación para América Latina y el Caribe (PRELAC) 11 al 13 de mayo. Santiago de Chile. http://www.elcorreo.eu.org/IMG/pdf/doc-1252. pdf

IIPE-UNESCO (2014): Políticas TIC en los sistemas educativos de América Latina. Informe sobre tendencias sociales y educativas en América Latina 2014. (Buenos Aires).

FERNANDEZ ENGUITA, M. y LEVIN, A. (1989): Las reformas comprensivas en Europa y las nuevas desigualdades educativas, en Revista de Educación, 289, (Madrid, MEC).

FERNANDEZ LAMARRA, N. y AGUERRONDO, I. (1990): La Planificación Educativa en América Latina, en AGUERRONDO, I. El Planeamiento Educativo como instrumento de cambio. (Buenos Aires, Ed. Troquel).

FRANCO, R. (1996): Los paradigmas de la política social en América Latina. Revista de la CEPAL, 58 (Santiago de Chile, CEPAL).

FUKUYAMA, F. (1992): El fin de la historia y el último hombre (Barcelona, Planeta)

FULLAN, M. (2002): Los nuevos significados del cambio en la educación (Barcelona: Octaedro) 
GAJARDO, M. (1999): Reformas educativas en América Latina: balance de una década. Doc. 15. (Santiago de Chile, PREAL)

GARRETON, M, A. (2002): La transformación de la acción colectiva en América Latina, Revista de la CEPAL, 76 (Santiago de Chile, CEPAL)

GRINDLE, M. (2000): La paradoja de la reforma educacional: Pronosticar el fracaso y encontrarnos con el avance, Serie Políticas. Formas y reformas de la educación, Año $2 \mathrm{~N}^{\circ} 6$, (PREAL, Santiago de Chile).

GURRIERI, A. (1987): Vigencia del Estado planificador en la crisis actual, Revista de la CEPAL, 31 (Santiago de Chile, CEPAL).

HOBBSBAWM, E. (1994): The age of extremes: The short twentieth century, 1914-1991. (Londres, Michael Joseph Ltd.).

HUSÉN, T. (1988): Investigación y políticas educativas. Una perspectiva internacional, en HUSÉN, T. Nuevo análisis de la sociedad del aprendizaje. (Madrid, Paidós/ MEC).

IAIES, G. (2011): Los debates de la política educativa en el nuevo milenio. (Buenos Aires, Aique Editorial).

IZCOVICH, G. (2013): La expansión educativa en el nivel inicial durante la última década. Cuaderno 16, (Buenos Aires, SITEAL-IIPE-UNESCO)

IZCOVICH, G. (2014): La expansión educativa en el nivel medio. América Latina 200o2010. Cuaderno 19, (Buenos Aires, SITEAL-IIPE-UNESCO).

KAUFMAN, R. y NELSON, J. (2005): Políticas de Reforma Educativa. Comparación entre países. Doc. No 33 (Santiago de Chile, PREAL).

KRAWCZY, N. (2002): La reforma educativa en América Latina desde la perspectiva de los organismos multilaterales», en Revista Mexicana de Investigación Educativa septiembre-diciembre, vol. 7, núm. 16 pp. 627-663. http://www.redalyc.org/ pdf/140/14001609.pdf

LANDSHERE, G. de (1996). La investigación educativa en el mundo. (México, FCE).

LEIRAS, M.; MALAMUD, A. y STEFANONI, P. (2016) ¿Porqué retrocede la izquierda? (Buenos Aires, Capital Intelectual).

LOPEZ, N. (2007) Las nuevas leyes de educación en América Latina: una lectura a la luz del panorama social de la región. (Buenos Aires, IIPE-UNESCO/Campaña Latinoamericana por el Derecho a la Educación).

MAHON, R. (2008): The post-bureaucracy shift: between path dependency, bricolage and translation: Commentary on the orientation 1 of the KNOW\&POL project, Report (http://knowandpol.eu)

MOURSHED, M., CHIJIOKE, C. y BARBER, M. (2012): Cómo continúan mejorando los sistemas educativos de mayor progreso en el mundo, MCKINSEY \& COMPANY, (Santiago de Chile, PREAL). 
NAVARRO, J.C. (2008): Los Sindicatos Docentes y las Políticas Educativas en América Latina, (Costa Rica, KAS-CEPP).

NAVARRO, J.C. (2006): Dos Clases de Políticas Educativas. La Política de las Políticas Públicas. (Santiago de Chile, Documento PREALC).

NAVARRO, J.C. (2007): Las reformas educativas como reformas del Estado: América Latina en las dos últimas décadas, en LORA, E. (edit) El estado de las reformas del Estado en América Latina. (Bogotá, BM-BID-Mayo Ediciones).

NOVOA, A. (1998): Professionnalisation des enseignants et sciences de l'education, Paedagogica Historica. International journal of the history of education, vol. III, pp. 403-430.

POPKEWITZ, Tomás (1991) Sociología política de las reformas educativas. (Madrid, Edit. Morata)

POGGI, Margarita (2011) Innovaciones educativas y escuelas en contextos de pobreza Evidencias para las políticas de algunas experiencias en América Latina. (Buenos Aires: IIPE-Unesco).

RAVELA, P. (2009): La evaluación del desempeño docente para el desarrollo de competencias profesionales, en MARTÍNEZRIZO, F.yMARTÍN, E. (coordinadores), Avances y desafíos en la evaluación educativa. Colección «Metas Educativas 2021, (Madrid, OEI-Fundación Santillana).

RIVAS, A. (2007): El desafio del derecho a la educación en Argentina: un dispositivo analítico para la acción. (Buenos Aires, CIPPEC).

RIVAS, A. (2015): América Latina después de PISA. Lecciones aprendidas de la educación en siete países (200O-2015). (Buenos Aires, CIPPEC-Natura-Instituto Natura)

RUIZ, G. (2016): Secondary Education in Five South American Countries. Revista Española de Educación Comparada, 27, 97-121.

SCHRIEWER, J. (1993): El método comparativo y la necesidad de externalización: criterios metodológicos y conceptos sociológicos, en SCHRIEWER y PEDRÓ (editores) Manual de Educación Comparada. Vol. II Teorías, Investigaciones, Perspectivas. (Barcelona, PPU).

SCHRIEWER, J. (2001) Formas de Externalização no Conhecimento Educacional. Cardernos PRESTIGE 5, (Lisboa, EDUCA)

SINTOMER, Y. (2017): ¿Condenados a la posdemocracia?, Revista Nueva Sociedad No 267, enero-febrero. (http://nuso.org/media/articles/downloads/1._TC_ Sintomer_267.pdf) Consultado 6 de octubre 2017

SITEAL (2016) Programas de Transferencias Condicionadas orientados a jóvenes. Notas sobre los avances y desafíos en 4 países de la región. Dato destacado 32, octubre. (Buenos Aires, IIPE-UNESCO) 
STEINER-KHAMSI, G. (Ed.) (2004): The Global Politics of Educational Borrowing and Lending. (New York, Teachers College Press).

SUASNÁBAR, C. (2004): Universidad e Intelectuales: educación y política en la Argentina (1955-1976). (Buenos Aires, Edit. Manantial / FLACSO)

SUASNÁBAR, C. (2013): Intelectuales, exilios y educación: producción intelectual e innovaciones teóricas en educación durante la última dictadura. (Rosario, Prohistoria Edit.)

TERIGI, F. (2007): Cuatro concepciones sobre el planeamiento educativo en la reforma educativa argentina de los noventa, Revista Archivos Analíticos de Políticas Educativas, Vol. 15, Nro 10 (Texas, Arizona State University).

VALLIANT, D. (2007): Planeamiento educativo en América latina: entre los desafios pendientes y los retos emergentes, (Buenos Aires, IIPE-UNESCO) 